\title{
THE DIMENSION OF PEAK-INTERPOLATION SETS
}

\author{
EDGAR LEE STOUT ${ }^{1}$
}

\begin{abstract}
The dimension of a peak-interpolation set in the boundary of a strongly pseudoconvex domain in $\mathbf{C}^{N}$ does not exceed $N-1$.
\end{abstract}

Recall that a peak set for a domain $D$ in $C^{N}$ is a subset $E$ of $b D$ for which there is $f \in A(D)$, i.e., an $f$ continuous on $\bar{D}$, holomorphic on $D$, with $f=1$ on $E$ and $|f|<1$ on $\bar{D} \backslash E$. The set $E$ is a peak-interpolation set for $D$ if given a nonzero $\varphi \in \mathcal{C}(E)$, there is $f \in A(D)$ with $f=\varphi$ on $E,|f|<\sup \{|\varphi(x)|: x \in E\}$ on $\bar{D} \backslash E$. For strongly pseudoconvex domains, a peak set is a peak-interpolation set. The general theory of these sets is given in [12].

Very little is known about the structure of peak-interpolation sets for domains in $\mathrm{C}^{N}$, but W. Rudin conjectured [11] that a peak-interpolation set for a strongly pseudoconvex domain in $\mathbf{C}^{N}$ has dimension not more than $N-1$. In [5] it was shown that this dimension cannot exceed $N$. The present paper is devoted to a proof of Rudin's conjecture: The correct bound is $N-1$. We obtain this result for a class of domains more extensive than the class of strongly pseudoconvex domains.

The idea of the proof, in $\mathbf{C}^{2}$, is that, as shown by Frankl and Pontrjagin [6] (cf. [7 and 2]), a closed, 2-dimensional subset of $\mathbf{R}^{3}$ disconnects some open connected subset of $\mathbf{R}^{3}$. (Curiously, this result seems not to have found a place in the modern texts on dimension theory.) In the case of domains in $\mathbf{C}^{N}$, we use a suitable cohomological generalization of this fact given in [10].

We should note explicitly that we understand dimension in the topological sense; the example of Tumanov [14] shows that there is no such result for metric dimension. [ADDED IN PROOF. A more definitive example has been obtained by B. S. Henriksen. She has constructed a peak-interpolation set of Hausdorff dimension $2 N-1$ in the boundary of a strongly pseudoconvex domain in $\mathbf{C}^{N}$. See Math. Ann. 259 (1982), 271-277.]

To formulate the result we prove, let us recall that a point $p$ in the boundary of a convex domain $\Delta$ is said to be rstrongly exposed if there are neighborhoods of $p$ in $b \Delta$ of arbitrarily small diameter and of the form $\{z \in b D: L(z)<0\}$ where $L$ is a real-valued, real affine functional on $\mathbf{C}^{N}$ with $L(p)=0$. Equivalently, it is possible to cut off from $\bar{\Delta}$ arbitrarily small neighborhoods of $p$ in $\bar{\Delta}$ with real hyperplanes. Each point in the boundary of the ball has this property as does each point in the distinguished boundary of the polydisc.

Received by the editors January 4, 1982.

1980 Mathematics Subject Classification. Primary 32E25.

'Research supported in part by Grant \# MCS 810768. 
THEOREM. Let $\Delta$ be a bounded open convex set in $\mathbf{C}^{N}$. If $F \subset b \Delta$ is a peak set that consists entirely of strongly exposed points, then $\operatorname{dim} F \leqslant N-1$.

This result implies the corresponding result for smoothly bounded, strongly pseudoconvex domains, for the question is local, and near each point in the boundary of a smoothly bounded strongly pseudoconvex domain $D, b D$ is strictly convex with respect to some set of local holomorphic coordinates.

Proof of TheOrem. Notice first that the set $F$ is necessarily polynomially convex. Fix a real hyperplane $\Pi$ in $\mathbf{C}^{N}$ that meets $\Delta$, and let $F^{+}$denote the intersection of $F$ with one of the closed halfspaces determined by $\Pi$. The set $S=(\Pi \cap \bar{\Delta}) \cup F^{+}$is easily seen to be polynomially convex: If $S$ differs from its polynomially convex hull, $\hat{S}$, choose $p \in \hat{S} \backslash S$, and let $\mu$ be a Jensen measure for $p$ supported on $S$. (For Jensen measures, see [4 or 13].) As $F$ is a peak set for $A(\Delta)$, there is $g \in A(\Delta)$ that vanishes identically on $F$ and that satisfies $g(p)=1$. From

$$
0=\log |g(p)| \leqslant \mu(\log |g|)
$$

we find that $\mu$ is concentrated on $\Pi \cap \bar{\Delta}$ whence, by convexity, $p \in \Pi \cap \bar{\Delta} \subset S$, a contradiction.

For the sake of clarity, we treat the case $N=2$ of our theorem separately from the case of general $N$. This not necessary on logical grounds, but it may shed some light on the result.

Assume then that $N=2$ and $\operatorname{dim} F=2$. By convexity, $b D$ is topologically equivalent to the three-sphere $S^{3}$. According to the result of Frankl and Pontrjagin cited above, there is a connected open set $U$ in $b D$ such that $U \backslash F$ is not connected. It follows, from the assumption that the points of $E$ are strongly exposed, that for some $p \in F$, there is a real hyperplane $\Pi$ that meets $\Delta$ and that passes so near $p$ that if $W$ is the component of $b \Delta \backslash \Pi$ containing $p$, then $W \backslash F$ is not connected. Thus, the compact, polynomially convex set $(\Pi \cap \bar{\Delta}) \cup(W \cap F)$ disconnects the topological three-sphere $(\Pi \cap \bar{\Delta}) \cup W=\Sigma$. The surface $\Sigma$ is the boundary of a convex domain, viz., one of the components, $\Delta^{+}$, of $\Delta \backslash \Pi$. According to a result of Alexander [1], no polynomially convex subset of $S^{2 N-1}=b B_{N}, B_{N}$ the unit ball in $C^{N}$, disconnects $S^{2 N-1}$. Alexander's proof applies verbatim when $B_{N}$ is replaced by an arbitrary bounded, open convex domain. Applied to $b \Delta^{+}=(\Pi \cap \bar{\Delta}) \cup W$ and the polynomially convex set $(\Pi \cap \bar{\Delta}) \cup(W \cap F)$, we see that we have a contradiction. This establishes the case $N=2$ of our Theorem.

We now take up the case of general $N$. Thus, assume $\Delta \subset \mathbf{C}^{N}$ and assume, for the sake of contradiction, that $\operatorname{dim} F=N$. According to Theorem 2 of [10, p. 7], there is a point $p \in F$ that has a neighborhood $U_{0}$ in $b \Delta$ with the property that for every open $V$ with $p \in V \subset U_{0}$, the natural map

$$
\iota_{V U_{0}}: H_{*}^{N}(V \cap F) \rightarrow H_{*}^{N}\left(U_{0} \cap F\right)
$$

is nonzero. (All of our cohomology groups have coefficients in the integers, but we shall surpress the coefficient group from the notation. The star denotes cohomology with compact supports.) 
Fix a real hyperplane $\Pi$ passing through $\Delta$ and missing $p$, $\Pi$ so close to $p$ that the component $W$ of $b \Delta \backslash \Pi$ containing $p$ is contained in $U_{0}$. The set $F^{\dagger}=(F \cap \bar{W}) \cup$ $(\Pi \cap \bar{\Delta})$ is polynomially convex, and so

$$
H^{k}\left(F^{\dagger}\right)=0, \quad k=N, N+1, \ldots
$$

(This is essentially due to Andreotti and Narasimhan [3]; for the rather formal deduction of the vanishing of these groups from what is written in [3], see [5].) The set $(\Pi \cap \bar{\Delta}) \cup W=\Sigma$ is homeomorphic to the sphere $S^{2 N-1}$; it is a convex surface. From the exact cohomology sequence [8, p. 190]

$$
\cdots \rightarrow H^{k}(\Sigma) \rightarrow H^{k}\left(F^{\dagger}\right) \rightarrow H_{*}^{k+1}\left(\Sigma \backslash F^{\dagger}\right) \rightarrow H^{k+1}(\Sigma) \rightarrow \cdots
$$

and the fact that $H^{r}(\Sigma)=0$ for $0<r<2 N-1$, we find

$$
H_{*}^{k+1}\left(\Sigma \backslash F^{\dagger}\right) \simeq H^{k}\left(F^{\dagger}\right)
$$

for $1 \leqslant k \leqslant 2 n-2$, and this yields

$$
H_{*}^{k+1}\left(\Sigma \backslash F^{\dagger}\right)=0, \quad k=N, N+1, \ldots, 2 N-2 .
$$

On the other hand, we have, by the choice of $W$, that

$$
H_{*}^{N}\left(W \cap F^{\dagger}\right)=H_{*}^{N}(W \cap F) \neq 0 .
$$

Consider the exact cohomology sequence

$$
\cdots \rightarrow H_{*}^{N}(W) \rightarrow H_{*}^{N}\left(W \cap F^{\dagger}\right) \rightarrow H_{*}^{N+1}\left(W \backslash F^{\dagger}\right) \rightarrow H_{*}^{N+1}(W) \rightarrow \cdots .
$$

As $W$ is homeomorphic to $\mathbf{R}^{2 N-1}$, we have $H_{*}^{N}(W)=0$ whence the nonzero group $H_{*}^{N}\left(W \cap F^{\dagger}\right)$ injects into the group $H_{*}^{N+1}\left(\mathrm{~W} \backslash F^{\dagger}\right)$. Since the sets $\Sigma \backslash F^{\dagger}$ and $W \backslash F^{\dagger}$ coincide, we have reached a contradiction to (1). Thus, $\operatorname{dim} F<N$.

Notice that the proof just given does not require that the set $F$ be a peak-interpolation set; it need only be a peak set. In the case of strongly pseudoconvex domains the two notions coincide, but it is not known that they do in the geometric setting of the theorem.

Corollary. If $\Delta$ is a bounded, convex domain in $\mathbf{C}^{N}$, and if $f$ is a nonconstant element of $A(\Delta)$ bounded by one in modulus, then the set $M=\{z \in b \Delta:|f(z)|=1\}$ has dimension no more than $N$ if it consists entirely of strongly exposed points.

Proof. By hypothesis, $f$ maps $\bar{\Delta}$ to the closed unit disc $\bar{U}$ in $\mathrm{C}$, and $M=f^{-1}(b U)$. According to the Theorem, $\operatorname{dim} F^{-1}(z) \leqslant N-1$ for all $z \in b U$, and so, as $\operatorname{dim} b U$ = 1, $\operatorname{dim} M \leqslant N$ follows from [9, Theorem V1.7].

For strongly pseudoconvex domains, this result was given in [5].

\section{REFERENCES}

1. H. Alexander, A note on polynomial hulls, Proc. Amer. Math. Soc. 33 (1972), 389-391.

2. P. Alexandroff, Dimensionstheorie. Ein Beitrag zur Geometrie der abgeschlossen Mengen, Math. Ann. 106 (1932), 161-238.

3. A. Andreotti and R. Narasimhan, A topological property of Runge pairs, Ann. of Math. (2) 76 (1962), 499-509. 
4. E. Bishop, Holomorphic completions, analytic continuation, and the interpolation of seminorms, Ann. of Math. (2) 78 (1963), 468-500.

5. T. Duchamp and E. L. Stout, Maximum modulus sets, Ann. Inst. Fourier (Grenoble) 31 (1981), $37-69$.

6. F. Frankl and L. Pontrjagin, Ein Knotensatz mit Anwendung auf die Dimensionstheorie, Math. Ann. 102 (1930), 785-789.

7. F. Frankl, Charakterisierung der $n-1$-dimensonalen abgeschlossen Mengen der $\mathbf{R}^{n}$, Math. Ann. 103 (1930), 784-787.

8. R. Godement, Théorie des faisceaux, Hermann, Paris, 1964.

9. H. Hurewicz and H. Wallman, Dimension theory, Princeton Univ. Press, Princeton, N. J., 1948.

10. V. I. Kuz'minov, Homological dimension theory, Russian Math. Surveys 23 (5) (1968), 1-45.

11. W. Rudin, Peak-interpolation sets of class $C^{1}$, Pacific J. Math. 75 (1978), 267-279.

12. Function theory in the unit ball of $\mathbf{C}^{N}$, Springer-Verlag, New York, Heidelberg and Berlin, 1980.

13. E. L. Stout, The theory of uniform algebras, Boyden and Quigley, Tarrytown-on-Hudson, N. Y., 1971.

14. A. E. Tumanov, A peak set for the disc algebra of metric dimension 2.5 in the three-dimensional unit sphere, Math. USSR Izv. 11 (1977), 353-359.

Department of Mathematics, University of Washington, Seattle, Washington 98195 